\title{
Economic Impact of Hospitalization Past Maximal Neurosurgical Inpatient Benefit
}

\author{
Cara M. Rogers ${ }^{1}$, Christopher M. Busch ${ }^{1}$, Joshua A. Cuoco ${ }^{1}$, Zev Elias ${ }^{1}$, Gary R. Simonds ${ }^{1}$ \\ 1. Neurosurgery, Carilion Clinic-Virginia Tech Carilion School of Medicine, Roanoke, USA
}

Corresponding author: Cara M. Rogers, cmrogers@carilionclinic.org

\section{Abstract \\ Background}

The unsustainable cost of healthcare in the United States has made it important for all healthcare professionals to examine their practices for wasteful spending and work to mitigate these costs. When neurosurgical patients remain hospitalized beyond the point of maximum inpatient benefit, this represents a potential source of healthcare economic waste.

\section{Objective}

The objective of this study was to determine the direct and indirect costs to a hospital system when neurosurgical patients remain hospitalized past the maximum inpatient benefit and identify targets to improve this potential wasteful spending.

\section{Methods}

We performed an extensive chart review of all patients admitted to our neurosurgical service from the months of July to October 2016, who had been deemed medically stable for discharge but remained in the hospital past their ideal date of discharge. We analyzed for significant trends in patient factors, procedural acuity, disposition, funding, and other factors that contributed to the delays in discharge.

\section{Results}

A total of 334 patients were admitted to the Carilion Clinic-Virginia Tech Carilion (CC-VTC) inpatient neurosurgery service, and 50 of these admissions (15\%) resulted in medically unnecessary prolonged hospitalizations. These patients were hospitalized for a total of 324 days past the dates of ideal discharge. Elective cases had the maximum number of prolonged hospitalizations, while the emergent cases had the maximum number of prolonged hospitalization days. Patients with private insurance had the shortest number of prolonged hospitalization days, and uninsured patients had the longest. Patients requiring disposition to a rehabilitation or a nursing facility remained in the inpatient setting for longer periods than those destined for home. The most common factors limiting appropriate discharge were related to bed availability at outside facilities, funding issues, and differing opinions on appropriate disposition. The medically unnecessary days accounted for $41 \%$ of the total hospitalization but accounted for only $12.9 \%$ of

Received 08/06/2018 Review began 08/14/2018 Review ended 10/27/2018 Published 11/10/2018

\section{๑) Copyright 2018}

Rogers et al. This is an open access article distributed under the terms of the Creative Commons Attribution License CC-BY 3.0., which permits unrestricted use, distribution, and reproduction in any medium, provided the original author and source are credited. the billable charges. The billable cost per medically necessary day was $\$ 17,326$ in comparison to a medically unnecessary day of $\$ 2,070$. Indirect costs were inferred from these patients utilizing beds and resources that could have been allocated to others with acute needs, given that our hospital is at capacity and on diversion, a significant percentage of the time.

\section{Conclusion}

Neurosurgical patients remaining hospitalized past their maximal inpatient benefit have a significant economic impact on a hospital system. Identifying patients who are at risk for prolonged hospitalizations may provide us with the targets for improvement to mitigate this healthcare economic waste.

Categories: Neurosurgery, Quality Improvement

Keywords: inpatient healthcare costs, economic waste, disposition delays, neurosurgery, socioeconomics, healthcare system, healthcare improvement

\section{Introduction}

With healthcare costs reaching unsustainable levels, it is imperative that we identify sources of wasteful spending and implement changes to eliminate this waste. Hospital costs constitute the largest component of healthcare spending and are therefore a prime target for improvement. A potential source of healthcare economic waste exists when patients remain hospitalized after they have reached the "maximum inpatient benefit." This is a commonly encountered occurrence during our neurosurgical service at Carilion ClinicVirginia Tech Carilion (CC-VTC). The goal of our study was to determine the financial impact of inappropriately prolonged hospitalizations on our neurosurgical service. We also aimed to identify the potential targets for improvement. 


\section{Materials And Methods}

We tracked all patients admitted to the CC-VTC neurosurgical service from the months of July to October 2016. Patients who were admitted to our neurosurgical service but subsequently transferred to the care of another service were excluded from the study. The census was reviewed by the rounding attending physician and team on a daily basis. Patients who were deemed medically stable for discharge, but remained in the hospital, were determined to have reached the "maximum inpatient benefit," and were included in the study. These patients were considered to have medically unnecessary prolonged hospitalization days.

Each patient who qualified for inclusion was then studied through a chart review. The date of ideal discharge was considered the date the patient was medically stable for discharge. The date of actual discharge was the date that the patient was eventually discharged from the hospital. The total days of hospitalization were calculated from the date of admission to the date of discharge. The total days of medically unnecessary hospitalization were calculated as the difference between the ideal and actual dates of discharge.

We recorded patient demographics including age, gender, acuity of presentation, primary payer status, and disposition destination. The acuity of presentation was recorded as elective, urgent, or emergent. Elective cases were defined as admissions scheduled in advance for non-emergent procedures. Urgent cases were defined as admissions for procedures to preserve the patient's life but did not require emergent surgery. Emergent cases were defined as admissions for emergency surgery. The primary payer status was categorized as medicare, medicaid, private insurance, or uninsured. Disposition destination was categorized as home, inpatient rehabilitation, or nursing facility. Home disposition was defined as patients being discharged to their private residence with or without home health services. Inpatient rehabilitation was defined as any facility intended for a provisional stay and required patients to participate in therapies for at least three hours daily. Nursing facilities were defined as institutions without minimum participation requirements for short or long-term admission. Each case was also analyzed for the primary barriers to appropriate discharge.

A one-way analysis of variance was conducted to determine if statistical significance was present between the acuity of presentation, the primary payer status, or the type of disposition and the number of prolonged hospitalization days. For groups that demonstrated statistical significance, a Tukey's honestly significant difference test was conducted to determine the specific subgroups that exhibited statistical difference.

Economic impact was measured as direct and indirect costs to the hospital system. To determine the direct cost, a cost analysis was performed by the Director of Cost Accounting for each patient included in the study. We determined the billable costs for the entire hospitalization and the billable costs for the days of medically unnecessary hospitalization. The indirect economic impact was considered as any negative effect of having patients fill beds unnecessarily, thereby limiting space for other admissions.

\section{Results}

During the four-month period between July 1 and October 31, 2016, a total of 334 patients were admitted to the CC-VTC neurosurgical inpatient service at the Roanoke Memorial Hospital. This included outpatient elective admissions and patients admitted from the emergency room. Fifty patients, or $15 \%$ of these admissions, resulted in medically unnecessary prolonged hospitalizations. There was a female predominance in the cohort with $58 \%$ females and $42 \%$ males. The range of patient ages was from 20 to 90 years of age with the average patient age being 63 years. The details of these cases are listed in Table 1 . These 50 patients were hospitalized for a total of 784 days, of which 324 days were past the dates of ideal discharge. The average length of total hospitalization was 15.68 days, with the average length of prolonged hospitalization being 6.48 days. Therefore, $41 \%$ of the days that these patients were hospitalized were deemed medically unnecessary.

\begin{tabular}{|c|c|c|c|c|c|c|}
\hline Neurosurgical Procedure & Age & Sex & $\begin{array}{l}\text { Prolonged } \\
\text { Days }\end{array}$ & Acuity & $\begin{array}{l}\text { Payer } \\
\text { Status }\end{array}$ & $\begin{array}{l}\text { Disposition } \\
\text { Destination }\end{array}$ \\
\hline Craniotomy for clipping of a ruptured aneurysm & 37 & Female & 27 & Emergent & Self-pay & SNF \\
\hline Coiling of a ruptured aneurysm & 56 & Female & 12 & Emergent & Private & IPR \\
\hline Craniotomy for clipping of a ruptured aneurysm & 38 & Female & 14 & Emergent & Medicaid & IPR \\
\hline Coiling of a ruptured aneurysm & 38 & Female & 7 & Emergent & Private & Home \\
\hline Craniotomy for subdural hematoma evacuation & 74 & Female & 6 & Emergent & Medicare & IPR \\
\hline Craniotomy for clipping of a ruptured aneurysm & 61 & Female & 5 & Emergent & Private & IPR \\
\hline $\begin{array}{l}\text { Biopsy of the vertebral body of a pathologic } \\
\text { compression fracture }\end{array}$ & 50 & Female & 5 & Urgent & Private & Home \\
\hline Cranial wound exploration for wound dehiscence & 63 & Female & 8 & Urgent & Private & SNF \\
\hline
\end{tabular}




\begin{tabular}{|c|c|c|c|c|c|c|}
\hline Craniotomy for tumor resection & 66 & Female & 2 & Urgent & Medicare & IPR \\
\hline Anterior-posterior cervical fusion for trauma & 60 & Female & 3 & Urgent & Private & IPR \\
\hline Stereotactic needle biopsy of an intracranial lesion & 90 & Female & 3 & Urgent & Medicare & Home \\
\hline T7-L1 fusion for trauma & 59 & Female & 2 & Urgent & Private & Home \\
\hline Craniotomy for resection of tumor & 68 & Female & 2 & Urgent & Medicare & IPR \\
\hline Craniotomy for resection of tumor & 55 & Male & 7 & Urgent & Private & IPR \\
\hline $\begin{array}{l}\text { Burr hole evacuation of acute on chronic subdural } \\
\text { hematoma }\end{array}$ & 90 & Female & 10 & Urgent & Medicare & IPR \\
\hline Craniotomy for resection of tumor & 68 & Female & 6 & Urgent & Medicare & IPR \\
\hline Craniotomy for resection of tumor & 48 & Male & 2 & Urgent & Medicaid & IPR \\
\hline $\begin{array}{l}\text { Cranial wound exploration for wound } \\
\text { infection/dehiscence }\end{array}$ & 54 & Male & 7 & Urgent & Medicaid & SNF \\
\hline Craniotomy for resection of tumor & 47 & Male & 2 & Urgent & Medicaid & SNF \\
\hline Craniotomy for resection of tumor & 56 & Female & 1 & Urgent & Self-pay & Home \\
\hline Cranial wound exploration for pseudomeningocele & 58 & Male & 4 & Urgent & Medicaid & SNF \\
\hline Craniotomy for resection of tumor & 76 & Male & 20 & Urgent & Medicare & IPR \\
\hline Cranioplasty & 49 & Male & 6 & Elective & Private & IPR \\
\hline Cranioplasty & 63 & Female & 4 & Elective & Private & IPR \\
\hline Anterior-posterior cervical fusion & 81 & Female & 7 & Elective & Medicare & Home \\
\hline Re-admitted for pain control status-post cervical fusion & 62 & Male & 12 & Elective & Medicaid & IPR \\
\hline Craniotomy for resection/fenestration of cyst & 54 & Male & 3 & Elective & Medicare & SNF \\
\hline Craniotomy for clipping of an unruptured aneurysm & 65 & Male & 8 & Elective & Private & IPR \\
\hline $\begin{array}{l}\text { L3-5 decompression and repair of cerebrospinal fluid } \\
\text { leak }\end{array}$ & 84 & Female & 7 & Elective & Medicare & SNF \\
\hline Cranioplasty & 20 & Male & 20 & Elective & Medicaid & IPR \\
\hline Craniotomy for clipping of an unruptured aneurysm & 62 & Male & 9 & Elective & Medicaid & SNF \\
\hline $\begin{array}{l}\text { Craniotomy for resection of a cerebellopontine angle } \\
\text { tumor }\end{array}$ & 65 & Female & 22 & Elective & Medicare & IPR \\
\hline Cervicothoracic decompression and fusion & 73 & Male & 2 & Elective & Medicare & IPR \\
\hline L3-5 transforaminal lumbar interbody fusion & 55 & Female & 3 & Elective & Medicaid & Home \\
\hline Ventriculoperitoneal revision & 70 & Female & 8 & Elective & Medicare & IPR \\
\hline C3-5 posterior decompression & 63 & Male & 5 & Elective & Private & IPR \\
\hline C3-6 anterior cervical decompression and fusion & 55 & Male & 2 & Elective & Self Pay & IPR \\
\hline L2-S1 decompression and fusion & 64 & Female & 1 & Elective & Private & SNF \\
\hline Cranioplasty & 79 & Female & 2 & Elective & Medicare & IPR \\
\hline L4-5 decompression & 83 & Female & 4 & Elective & Medicare & SNF \\
\hline L4-5 decompression & 76 & Male & 3 & Elective & Medicare & IPR \\
\hline Resection of posterior cervical paraspinal mass & 75 & Male & 4 & Elective & Medicare & Home \\
\hline Revision C1-6 posterior fusion & 76 & Female & 3 & Elective & Medicare & SNF \\
\hline Craniotomy for clipping of an unruptured aneurysm & 53 & Female & 4 & Elective & Medicaid & Home \\
\hline C3-6 anterior cervical decompression and fusion & 77 & Male & 2 & Elective & Medicare & SNF \\
\hline C5-T1 posterior decompression and fusion & 74 & Male & 2 & Elective & Private & SNF \\
\hline L4-5 transforaminal lum & 60 & Female & 5 & Elective & Medicaid & Home \\
\hline
\end{tabular}




\section{Cureus}

\begin{tabular}{llllllll}
\hline L4-5 transforaminal lumbar interbody fusion & 73 & Male & 15 & Elective & Medicare & SNF \\
Craniotomy for clipping of an unruptured aneurysm & 66 & Female & 2 & Elective & Medicare & Home \\
L2-S1 decompression & 62 & Male & 4 & Elective & Private & SNF
\end{tabular}

TABLE 1: Details of cases that resulted in prolonged days of stay

SNF: skilled nursing facility; IPR: in-patient rehabilitation

Evaluation of the acuity of presentation in this cohort demonstrated that the elective cases had the greatest number of prolonged hospitalizations, while the emergent cases had the maximum prolonged hospitalization days. Of the 50 patients included in the study, there were six emergent, 16 urgent, and 28 elective cases. Elective cases accounted for $56 \%$ of prolonged hospitalizations. The elective patients remained in the hospital an average of 6.00 days past their ideal dates of discharge (Table 2). Urgent cases accounted for $32 \%$ of the prolonged hospitalizations and had an average of 5.25 days of medically unnecessary hospitalization. Emergent cases accounted for $12 \%$ of the prolonged hospitalizations and had an average of 11.83 days of medically unnecessary hospitalization. There was a significant effect of neurosurgical acuity on the number of prolonged hospitalization days at the $p<0.05$ level for the three conditions $[\mathrm{F}(2,47)=3.285, p=0.046]$. Post-hoc comparisons using the Tukey's honestly significant difference test indicated statistical significance between the emergent and urgent groups $(p=0.043)$ but did not demonstrate statistical significance between the emergent and elective groups $(p=0.062)$ or the elective and urgent groups $(p=0.894)$.

\begin{tabular}{|c|c|c|c|}
\hline Acuity & Patients & Prolonged Days & Average Prolonged Days \\
\hline Emergent & 6 & 71 & 11.83 \\
\hline Urgent & 16 & 84 & 5.25 \\
\hline Elective & 28 & 169 & 6.00 \\
\hline
\end{tabular}

TABLE 2: Prolonged days of stay based on acuity of presentation

Analysis of the primary payer status revealed that patients with private insurance had the shortest prolonged hospitalization days, and uninsured patients had the longest. Of the 50 patients included in the study, 15 had private insurance, 21 had medicare, 11 had medicaid, and three were uninsured. The average length of prolonged hospitalization was 5.27 days for patients with private insurance, 6.33 days for medicare patients, 7.45 days for medicaid patients, and 10.00 days for uninsured patients (Table 3). No statistical significance was observed between the type of insurance and the number of prolonged hospitalization days $(p=0.567)$.

\begin{tabular}{|c|c|c|c|}
\hline Payer Status & Patients & Prolonged Days & Average Prolonged Days \\
\hline Self Pay & 3 & 30 & 10.00 \\
\hline Medicaid & 11 & 82 & 7.45 \\
\hline Medicare & 21 & 133 & 6.33 \\
\hline Private & 15 & 79 & 5.27 \\
\hline
\end{tabular}

TABLE 3: Prolonged days of stay based on payer status

The length of medically unnecessary prolonged hospitalization also varied with the disposition plan. Patients requiring disposition to a rehabilitation or a nursing facility remained in the inpatient setting for a longer period than those destined for a private residence. Of the 50 patients included in the study, 11 were discharged home, 15 to nursing facilities, and 24 to inpatient rehabilitation. The average length of prolonged hospitalization was 3.91 days for patients discharged home, 6.53 days for patients discharged to a nursing facility, and 7.63 days for patients discharged to inpatient rehabilitation (Table 4). No statistical significance was observed between the type of disposition and the number of prolonged hospitalization days $(p=0.215)$. 


\section{Cureus}

\begin{tabular}{|c|c|c|c|}
\hline Disposition Destination & Patients & Prolonged Days & Average Prolonged Days \\
\hline Home & 11 & 43 & 3.91 \\
\hline SNF & 15 & 98 & 6.53 \\
\hline IPR & 24 & 183 & 7.63 \\
\hline
\end{tabular}

TABLE 4: Prolonged days of stay based on disposition destination

The barriers we encountered to discharging patients on their ideal dates of discharge were multifactorial in most cases. The most common factors limiting appropriate discharge were related to bed availability at outside facilities, funding issues, and differing opinions on appropriate disposition. Limited bed availability at inpatient rehabilitation and nursing facilities contributed to a delay in discharge in $54 \%$ of cases. Awaiting insurance authorization for inpatient rehabilitation and nursing facilities contributed to a delay in discharge in $20 \%$ of cases. A lack of adequate funding or benefits for inpatient rehabilitation and nursing facilities contributed to delays in $22 \%$ of cases. Multiple insured patients did not have benefits or had run out of their benefit for rehabilitation or nursing facility placement. Disagreement regarding appropriate disposition contributed to a delay in discharge $32 \%$ of cases. This discrepancy was oftentimes between our inpatient therapy consultant recommendations and the outpatient facility assessment of the patient needs. This included cases where the patients were felt to be too high functioning or too low functioning for disposition to the intended facility.

The direct cost of these medically unnecessary prolonged hospitalizations was determined through a cost analysis by the Director of Cost Accounting. The total billable charges for the hospitalizations of the 50 patients included in the study were $\$ 6,124,676$. The total billable charges for the medically unnecessary days past the ideal dates of discharge were $\$ 474,230$. The medically unnecessary days accounted for $41 \%$ of the total hospitalization but accounted for only $12.9 \%$ of the billable charges. The billable cost per medically necessary day was calculated to be $\$ 17,326$ in comparison to the billable cost per medically unnecessary day being $\$ 2,070$.

The indirect costs to the hospital system were not financially measurable but were inferred from the fact that these patients were filling hospital beds that could have been utilized for other patients. In general, our system remains at or near capacity for inpatient beds. Our hospital was on some level of diversion $42.5 \%$ of the shifts during this four-month period (Figure 1). The hospital is unable to accept transfers from the referring facilities or direct admissions from the outpatient setting when we are at capacity for the requested level of patient care. The patients included in this study were taking up medical/surgical unit floor beds during the medically unnecessary days of prolonged hospitalization. The medical/surgical floor beds were filled to capacity $31.25 \%$ of shifts on average during these months (Figure 2). Our emergency room also goes onto diversion when there is an increase in patient volumes that exceed the available resources. Our emergency room was on diversion a total of 238 hours in these four months, which is almost 10 full days or $8 \%$ of the time.

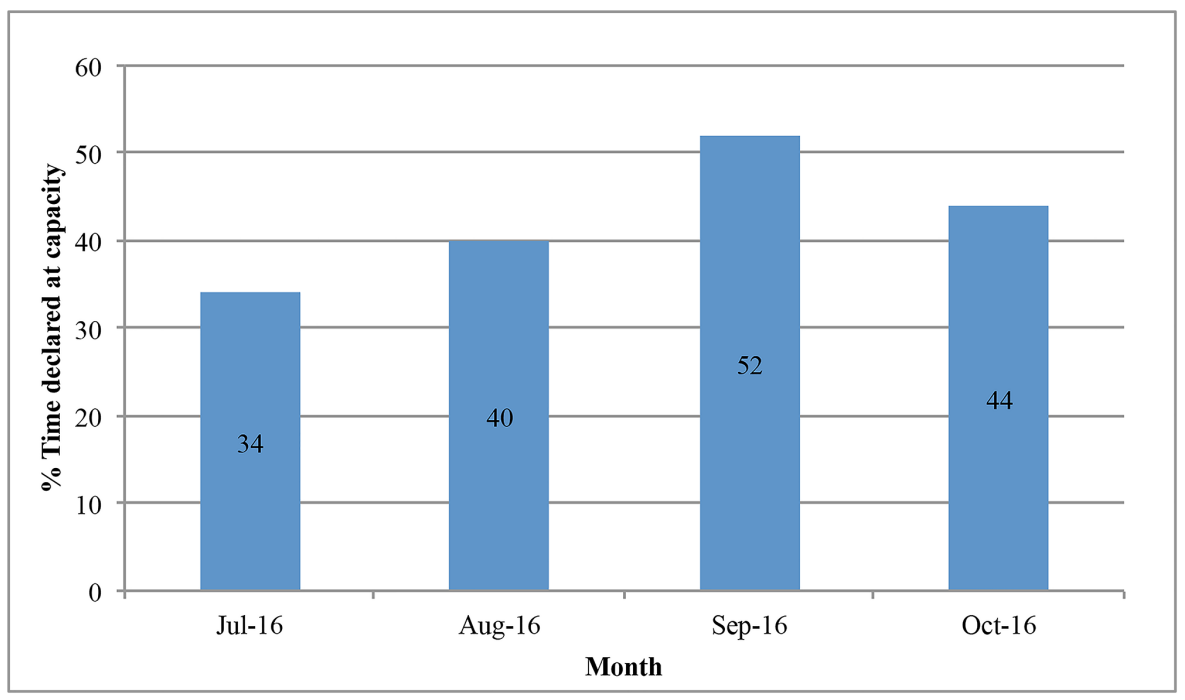

FIGURE 1: Percentage of time at declared capacity per shift 


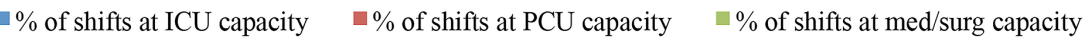

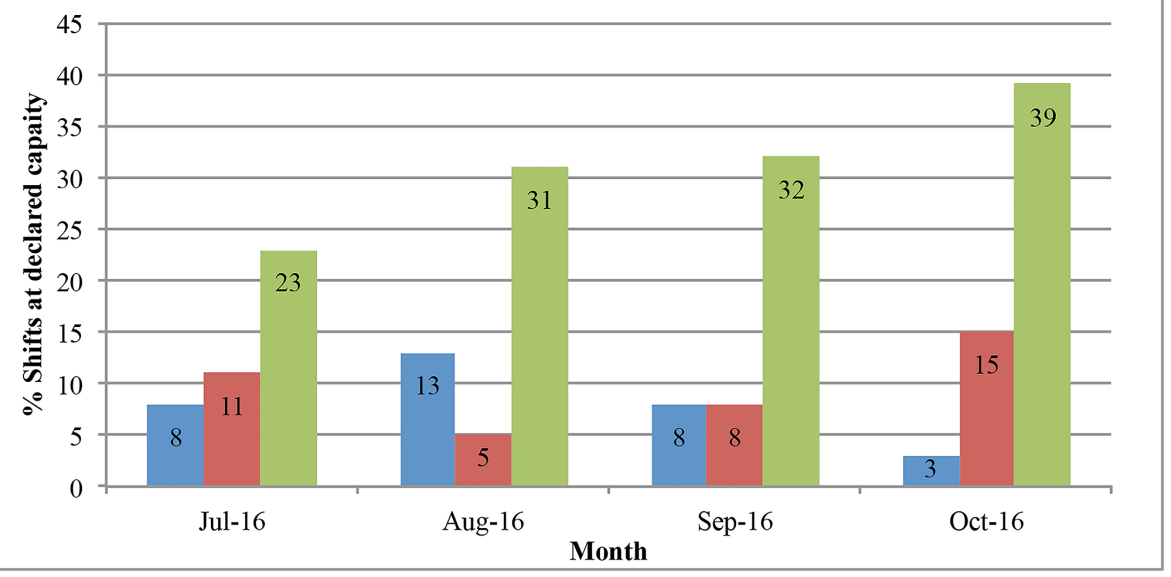

FIGURE 2: Percentage of shifts at declared capacity by the level of care

ICU: intensive care unit; PCU: progressice care unit

\section{Discussion}

The cost of healthcare in the United States has risen to an unsustainable level, which has forced us to evaluate the efficiency of utilization of these resources. Healthcare costs have been on a steady rise, accounting for 17.8 percent of the gross domestic product in 2015 with projections that it will continue to rise [1-2]. The national healthcare expenditure increased $5.8 \%$ that year to $\$ 3.2$ trillion dollars with the hospital expenditures increasing 5.6\% to $\$ 1$ billion dollars [1,3]. However, despite spending almost twice as much per capita on health care as other industrialized nations, according to the Census Bureau, the US ranks $42^{\text {nd }}$ in the world for life expectancy and has higher rates of infant mortality, obesity, and avoidable deaths than the global averages [4]. Therefore, even though the government continues to allocate a substantial and an increasing portion of our resources to healthcare, there has been no compensatory improvement in outcomes according to the Organization for Economic Cooperation and Development [5].

Concern exists that an unacceptable portion of healthcare spending is unnecessary, inefficient, or underutilized [4,6-7]. It was calculated by Pricewaterhouse Coopers in 2008 that 1.2 trillion dollars, over half of the healthcare costs that year, was wasteful spending [8]. They defined healthcare waste as "costs that could have been avoided without a negative impact on quality” [8]. In order to eliminate this waste, it is essential for providers to identify sources of this wasteful spending. Inpatient hospital costs are of particular importance because they make up the largest component of healthcare spending and represent a prime target for improvement.

Our neurosurgical team at CC-VTC in Roanoke, Virginia, identified medically unnecessary prolonged hospitalizations as a potential source of wasteful spending. In a four-month period, $15 \%$ of the patients admitted to our service had medically unnecessary prolonged hospitalizations. The average length of prolonged hospitalization was 6.48 days and accounted for $41 \%$ of the days that these patients were hospitalized. The average billable cost per medically unnecessary day was $\$ 2,070$ which compounds on itself for every day that patients remain hospitalized needlessly. This resulted in $\$ 474,230$ of billable costs that were unnecessary and did not improve the quality of care.

While this represents a wasteful utilization of resources with respect to the healthcare system, the impact on the hospital system is also substantial. There are predetermined bundled costs for patients being admitted for a particular neurosurgical procedure after which the services that a hospital can charge for diminishes precipitously regardless of disposition delays. This explains why the billable cost for each medically necessary day is over eight times greater than the cost for each medically unnecessary day. The financial impact of that difference is likely great. The hospital was on some level of diversion $42.5 \%$ of the time, and the emergency room was on diversion $8 \%$ of the time during this four-month period. Therefore, while patients remained in our hospital beds past their ideal dates of discharge, this limited our ability at times to bring in patients with acute medical needs. While the hospital system is only able to charge a comparatively small amount of money per medically unnecessary day, they are losing out on a greater potential revenue from these diverted new admissions. This represents a potential loss of profit for the hospital system and also a potential delay in care for patients.

We were able to identify the potentially preventable causes of prolonged hospitalizations that represent 
targets for improvement. Notably, we found that $56 \%$ of prolonged hospitalizations occurred in our elective admissions. In $50 \%$ of these cases, there were delays due to the lack of bed availability at the rehabilitation or nursing facility of the patients choosing. In $22 \%$ of these cases, there were delays while we awaited insurance authorization for these facilities. This may be preventable by identifying patients who are unlikely to be discharged home and setting up their disposition prior to admission. This could include determining the insurance benefits they have, preference of facilities, bed availability at these facilities, and securing insurance authorization. Patients admitted for urgent procedures would likely benefit from this preadmission process as well. Patients admitted for emergent procedures should have social workers involved early in their admissions to identify the potential barriers to discharge. This would include identifying legal decision makers, funding sources, and viable discharge possibilities. We also identified that there is a shortage of beds at the rehabilitation and nursing facilities in our region, resulting in delays in discharge in $54 \%$ of the cases. It may, therefore, be beneficial for our hospital system to work toward increasing the available beds at the current facilities or increase the number of facilities in the region.

\section{Conclusions}

Our results confirmed that unnecessarily prolonged hospitalizations on a neurosurgical service have a significant impact on the medical system. It is a prime example of unnecessary and inefficient utilization of resources to keep patients hospitalized without inpatient medical needs. It contributed to significant bed shortages, which forces us to turn away patients with medical needs. By identifying the commonly encountered barriers to appropriate discharge, we have now discovered several targets for improvement.

\section{Additional Information}

\section{Disclosures}

Human subjects: Consent was obtained by all participants in this study. Animal subjects: All authors have confirmed that this study did not involve animal subjects or tissue. Conflicts of interest: In compliance with the ICMJE uniform disclosure form, all authors declare the following: Payment/services info: All authors have declared that no financial support was received from any organization for the submitted work. Financial relationships: All authors have declared that they have no financial relationships at present or within the previous three years with any organizations that might have an interest in the submitted work. Other relationships: All authors have declared that there are no other relationships or activities that could appear to have influenced the submitted work.

\section{References}

1. Dieleman JL, Squires E, Bui AL, et al.: Factors associated with increases in US health care spending, 19962013. JAMA. 2017, 318:1668-1678. 10.1001/jama.2017.15927

2. Dieleman JL, Baral R, Birger M, et al.: US spending on personal health care and public health, 1996-2013. JAMA. 2016, 316:2627-2646. doi:10.1001/jama.2016.16885

3. Rotarius T, Liberman A: Health care affordability: how to make it a reality . Health Care Manag (Frederick). 2015, 34:288-292.

4. Hood VL, Weinberger SE: High value cost-conscious care: an international imperative . Eur J Intern Med. 2012, 23:495-498. 10.1016/j.ejim.2012.03.006

5. Squires D, Anderson C: U.S. health care from a global perspective: spending, use of services, prices, and health in 13 countries. Issue Brief (Commonw Fund). 2015, 15:1-15.

6. Keehan SP, Cuckler GA, Sisko AM, et al.: National health expenditure projections, 2014-24: spending growth faster than recent trends. Health Aff (Millwood). 2015, 34:1407-1417. 10.1377/hlthaff.2015.0600

7. Swensen SJ, Kaplan GS, Meyer GS, et al.: Controlling healthcare costs by removing waste: what American doctors can do now. BMJ Qual Saf. 2011, 20:534-537. 10.1136/bmjqs.2010.049213

8. The price of excess: identifying waste in healthcare spending . (2008). Accessed: November 23, 2017 : http://www.oss.net/dynamaster/file_archive/080509/59f26a38c114f2295757bb6be522128a/The\%20Price\%20of\%20Excess\%2 \%20Id.... 